\title{
Relação dos Sintomas Otológicos nas Disfunções Temporomandibulares
}

\section{Relation of the Otological Symptoms in the Temporomandibular Dysfunctions}

\section{Ilza Maria Machado*, Paulo Roberto Pialarissi**, Thainá Decicco Minici***,Juliana Rotondi***, Léslie Piccolotto Ferreira ${ }^{* * * *}$.}

\author{
* Mestre. Fonoaudiologa \\ *** Doutor. Médico Otorrinolaringologista. \\ **** Graduação. Fonoaudióloga. \\ ***** Doutora. Professora Titular do Departamento de Fundamentos da Fonoaudiologia da PUC-SP; Coordenadora e docente do Curso de Especialização em Fonoaudiologia \\ -VOZ-PUC-SP/COGEAE. \\ Instituição: $\quad$ Pontifícia Universidade Católica de São Paulo - PUC/SP \\ São Paulo / SP - Brasil \\ Endereço para correspondência: Ilza Maria Machado - Rua Napoleão de Barros 1058 - Apto.11 - Bairro: Vila Clementino - São Paulo / SP - Brasil - CEP: 04024-003 - \\ Telefone: (+55 11) 9421-4049 - E-mail: ilzamachado@yahoo.com.br \\ Artigo recebido em 24 de Março de 2010. Artigo aprovado em 27 de Abril de 2010
}

RESUMO

Introdução:

As disfunções da articulação temporomandibular (ATM) abrangem uma série de problemas clínicos que envolvem a musculatura mastigatória, a própria articulação e estruturas associadas à região da orelha.

Objetivo:

Método:

Analisar a relação entre disfunção temporomandibular e sintomas otológicos.

Fizeram parte do estudo retrospectivo 20 mulheres com média de idade de 22,38 anos. O estudo foi realizado por uma equipe multidisciplinar que envolveu médico otorrinolaringologista, fonoaudiólogas e ortodontista. As participantes foram submetidas ao preenchimento de um questionário que abordou questões sobre sintomas otológicos e posteriormente foram submetidas a um exame otoscópico e exame clínico da articulação temporomandibular.

Resultados: $85 \%$ da amostra tiveram disfunção temporomandibular conjuntamente com queixas de sintomas otológicos. Os sintomas otológicos mais autorreferidos foram de plenitude auricular (50\%), zumbido $(35 \%)$ e vertigem (10\%)

Conclusão:

Os resultados mostraram alto percentual entre as DTMs e sintomas otológicos. Outras pesquisas com uma amostra maior são sugeridas com o intuito de confirmar a correlação entre DTM e Sintomas otológicos.

Palavras-chave: transtornos da articulação temporomandibular, transtornos da audição, dor facial.

\section{SUMMARY}

Introduction:

The temporomandibular joint dysfunctions (TMJ) include several clinical problems which wrap the masticatory muscle, the joint itself and structures associated to the ear region.

Objective:

Method:

To analyze the relation between temporomandibular dysfunction and otological symptoms.

Were part of a retrospective study 20 women with mean age of 22, 38 years. This study was realized by a multidisciplinary team that wrapped otorhinolaryngologist, speech therapist and orthodontist. The participants were submitted to the filling of a questionnaire that addressed questions about otological symptoms and later they were submitted to an otoscopy exam and a clinical examination of the temporomandibular joint.

Results:

Eighty-five per cent had simultaneously temporomandibular dysfunction with otological symptoms complaints. The otological symptoms more mentioned were auricular plenitude (50\%), tinnitus (35\%), and dizziness (10\%).

Conclusion:

The results showed high percentage between DTMs and otological symptoms. Other researches with a larger sample are suggested with the aim of confirm the correlation between DTM and otological symptoms.

Keywords: temporomandibular joint disorder, hearing disorders, facial pain. 


\section{INTRODUÇÃO}

A articulação temporomandibular (ATM) é considerada uma das mais completas do ser humano, pois permite diversos movimentos, como: abertura, fechamento, protrusão, retrusão e lateralidade da mandíbula (1). As disfunções temporomandibulares (DTMs) abrangem uma série de problemas clínicos que envolvem a musculatura mastigatória, a própria articulação e estruturas associadas $(2,3,4,5)$.

Estudos demonstram $(6,7,8,9)$ a não existência de um fator etiológico que se responsabilize pela DTM, sendo, portanto, uma doença de caráter multifatorial, que inclui fatores traumáticos, problemas degenerativos, hábitos nocivos, posição anormal do côndilo e do disco articular, atividades excessivas da musculatura mastigatória e variáveis psicossociais e psicológicas.

Cerca de 70 a 93\% da população, em geral, apresenta pelo menos um sinal e/ou sintoma de DTM, mas apenas uma, em cada quatro pessoas, tem tal conhecimento (2, 10). Estudo realizado com o objetivo de verificar a sintomatologia das DTMs em 144 indivíduos (10) ressalta que somente 5 a 13\%, com mais de um sinal e/ou sintoma de DTM, apresentam alteração clinicamente significativa, como dor ou graves disfunções.

As DTMs são caracterizadas por uma série de sinais e sintomas clínicos que se manifestam, como: dores musculares, cefaleia, estalidos na ATM, dificuldades em realizar movimentos mandibulares, limitação na amplitude dos movimentos mandibulares, especialmente com relação à abertura bucal $(4,11,12,13,14,15,16,17,18)$.

Esses sinais advêm de inflamações da área retrodiscal, uma vez que o disco articular encontra-se deslocado (6). A área retrodiscal ao sofrer tensão pode produzir inflamação que resulta em dor com característica contínua (19).

No que se refere aos sintomas otológicos relacionados às DTMs, os mais citados pela literatura $(20,21)$, são os de zumbido, otalgia, plenitude auricular, perda de audição e vertigem.

A associação das DTMs e a origem dos sintomas otológicos, ainda, não estão totalmente esclarecidas ( 4,22 , 24, 24). Existem algumas hipóteses sobre a relação entre sintomas otológicos e as DTMs. A primeira delas é a de que o mau posicionamento do côndilo da mandíbula poderia ocasionar sintomas de otalgia, zumbido e vertigem.

O zumbido em indivíduos com DTM pode ser decorrente de um sinal neural, induzido pelo nervo aurículo temporal ou por redução dos sinais sensórias (2).
Este sintoma pode ser decorrente de outras alterações, como doenças da orelha interna ou sistema nervoso central (25).

Um caso repórter sobre trauma na ATM (26) menciona que o côndilo da mandíbula ao sofrer um deslocamento pode provocar um estiramento da cadeia ossicular e desencadear sintomas aurais.

Outra hipótese vai na direção de apontar que a ocorrência de hiperatividade nos músculos da mastigação pode contrair o músculo tensor do tímpano e a membrana timpânica, fato que resulta em uma disfunção tubário com sintomas de plenitude auricular, desequilíbrio e perda de audição $(20,24,26,27)$.

Este estudo, portanto, tem como objetivo analisar a relação entre disfunção temporomandibular e sintomas otológicos.

\section{MÉTOdO}

Esta pesquisa foi aprovada pelo Comitê de Ética em pesquisa, sob o $\mathrm{n}^{\circ}$. 04/803, e todos os participantes assinaram termo de consentimento livre e esclarecido.

Participaram do estudo 20 alunas graduandas em Fonoaudiologia, com média de idade 22,38 anos, que previamente haviam realizado testes de audição, ou seja, audiometria tonal, logoaudiometria e imitanciometria.

Todas as participantes foram avaliadas por uma equipe multidisciplinar que incluiu médico otorrinolaringologista, fonoaudiólogas e uma ortodontista. As participantes do estudo foram submetidas ao preenchimento de um questionário, entregue pelas fonoaudiólogas, que contou com questões relacionadas aos sintomas de DTM e otológicos, elaborado para esta pesquisa.

No laboratório de anatomia da própria universidade, foi realizado exame de otoscopia com o médico otorrinolaringologista para verificar presença (quando presente fluido visível na orelha média, opacificação, retração e imobilidade de membrana timpânica na insuflação) ou ausência (íntegra, translúcida e com movimentação na insuflação) de alteração.

Foi realizado exame clínico da articulação temporomandibular por ortodontista, que levantou dados referentes à presença de DTM. Verificou-se presença de dor na musculatura da mastigação, sinais clínicos de DTM (crepitação, estalido, desvio dos movimentos mandibulares) e presença de hábitos deletérios (bruxismo, apertar os dentes e onicofagia). 
Para análise estatística foram considerados os seguintes dados: no questionário foi considerado sujeito com alteração otológica aquele que autorreferiu pelo menos um sintoma de otalgia, plenitude auricular, zumbido e vertigem: e na avaliação odontológica, a DTM foi definida pela presença de três ou mais sinais e/ou sintomas, sendo imprescindível o sintoma de dor.

Todos os dados foram digitados na planilha Excel e para medir o grau de relação entre sintomas otológicos e DTM foi utilizado o Teste Exato de Fisher, e o nível de significância adotado, de 5\%.

\section{RESULTADOS}

A Tabela 1 apresenta os dados levantados a partir da avaliação odontológica.

A Tabela 2 apresenta os dados levantados a partir do questionário e caracterizam os participantes quanto aos aspectos relacionados aos sintomas otológicos.

A Tabela 3 apresenta a descrição dos movimentos mandibulares e assimetria de face na avaliação da motricidade orofacial.

Tabela I. Distribuição numérica e percentual da avaliação odontológica que avaliou aspectos relacionados à presença de DTM $(n=20)$.

\begin{tabular}{llcccc}
\hline & VARIÁVEL & \multicolumn{2}{c}{ Ausência } & \multicolumn{2}{c}{ Presença } \\
& & $\mathrm{n}$ & $\%$ & $\mathrm{n}$ & $\%$ \\
\hline Presença de DTM $(\mathrm{n}=20)$ & & 3 & 15 & 17 & 85 \\
Sintoma de dor & Dor de cabeça & 8 & 40 & 12 & 60 \\
Presença de dor a pal pação & Músculo pterigóideo lateral & 5 & 25 & 15 & 75 \\
& Dorna ATM & 8 & 40 & 12 & 60 \\
& Músculo pterigóideo medial & 8 & 40 & 12 & 60 \\
& Músculo masseter & 12 & 60 & 8 & 40 \\
& Músculo temporal & 15 & 75 & 5 & 25 \\
& Músculo digastrico & 17 & 85 & 3 & 15 \\
& Músculo occipital & 17 & 85 & 3 & 15 \\
& Músculo trapézio & 17 & 85 & 3 & 15 \\
& Músculo esternocleidomastoideo & 17 & 85 & 3 & 15 \\
Sinais de DTM & Músculo peitoral & 19 & 95 & 2 & 10 \\
& Estalo na ATM & 6 & 30 & 14 & 70 \\
& Desvio mandibular & 8 & 40 & 12 & 60 \\
Hábitos deletérios & Mastigação unilateral & 12 & 60 & 8 & 40 \\
& Apertamento dental & 10 & 50 & 10 & 50 \\
& Bruxismo & 14 & 70 & 6 & 30 \\
& Unicofagia & 17 & 85 & 3 & 15 \\
\hline
\end{tabular}

Tabela 2. Distribuição numérica e percentual das respostas ao questionário que levantou aspectos aos sintomas otológicos $(n=20)$.

\begin{tabular}{llcccc}
\hline & VARIÁVEL & \multicolumn{2}{c}{ Ausência } & \multicolumn{2}{c}{ Presença } \\
& & $N$ & $\%$ & N & $\%$ \\
\hline Sintomas otológicos $(\mathrm{n}=20)$ & & 5 & 25 & 14 & 75 \\
Sintomas & & 10 & 50 & 10 & 50 \\
& Plenitude auricular & 13 & 65 & 7 & 35 \\
& Zumbido & 18 & 90 & 2 & 10 \\
& Vertigem & 19 & 95 & 1 & 5 \\
& Otalgia & 19 & 95 & 1 & 5 \\
\hline
\end{tabular}


Tabela 3. Correlação entre sintomas otológicos e disfunção temporomandibular $(n=20)$.

\begin{tabular}{llccccccc}
\hline & & \multicolumn{3}{c}{ Sintomas otológicos } & \multicolumn{2}{c}{ Total } \\
& & Ausência & Presença & & \\
& $N$ & $\%$ & $N$ & $\%$ & $N$ & $\%$ \\
\hline Disfunção Temporomandibular & Ausência & 2 & 40,0 & 1 & 6,7 & 3 & 15,0 \\
& Presença & 3 & 60,0 & 14 & 93,3 & 17 & 85,0 \\
\hline & Total & 5 & 100,0 & 15 & 100,0 & 20 & 100,0 \\
\hline
\end{tabular}

Teste exato de Fisher $\mathrm{p}$-valor $=0,1404$

Na Tabela 3 foram explicitadas a relação e concordância entre os sintomas otológicos e DTM.

\section{DISCUSSÃO}

Este estudo buscou uma possível relação entre disfunção temporomandibular e sintomas otológicos. Foi constatado em $85 \%$ da amostra presença de dor acompanhado de dois ou mais sinais e/ou sintomas para DTM. As DTMs são caracterizadas por diversos sinais e sintomas que se manifestam na musculatura orofacial $(2,3,4,5)$, e região pré-auricular, próxima ao ouvido (21).

Os principais sinais e sintomas de DTM encontrados neste estudo foram: estalo (70\%), desvio dos movimentos mandibulares (60\%), dor de cabeça (60\%) e dor sobre a ATM (60\%). Estudo constatou-se, em indivíduos com DTM, presença de desvio da mandíbula e redução vertical dos movimentos mandibulares (18). Estes problemas podem ser desencadeados por fatores que envolvem a oclusão, posição anormal do côndilo da mandíbula, atividade excessiva da musculatura orofacial, hábitos parafuncionais e variáveis psicossociais e psicológicas (7).

Foi observado dor a palpação na musculatura da mastigação, principalmente, no músculo pterigóideo lateral $(75 \%)$. Isso pode estar relacionado ao fato de os músculos pterigóideos, estabelecerem relação direta com o côndilo da mandíbula e trabalharem conjuntamente com os músculos masseteres e temporais, no desempenho dos movimentos mandibulares (27). Estudo (6) menciona que a dor advém de inflamações ou tensão na área articular.

Os resultados do presente estudo mostram que não houve correlação estatística entre sintomas otológicos e DTM (p-valor $=0,1404)$. Dos 20 pacientes analisados 17 (85\%) tiveram DTM e 15 (75\%) sintomas otológicos. Destes 15 pacientes com sintomas otológicos, 14 (93, 3\%) apresentaram DTM. Assim, no grupo de pacientes com DTM (17), 85\% também autorreferiram presença de sintomas otológicos.
Os sintomas otológicos mais autorreferidos pela amostra foram de plenitude auricular e zumbido. Estes sintomas também têm sido relatados pela literatura em pacientes com DTM $(20,23,24,26,27)$. Os sintomas otológicos estão associados com dificuldades nos movimentos mandibulares, abrir a boca e nas funções do sistema estomatognático (23) (mastigação, deglutição, respiração e fala).

É importante focar que se deve ficar atento ao sintoma de zumbido, visto que tal alteração pode ser decorrente de doenças da orelha interna ou sistema nervoso central e não apenas pela DTM (25).

Pesquisa (26) aponta que o côndilo da mandíbula ao sofrer um deslocamento pode provocar estiramento da cadeia ossicular e, assim, a ocorrência de sintomas otológicos.

Outra hipótese vai na direção de apontar que a ocorrência de hiperatividade nos músculos da mastigação, por sua vez, levará a contração do músculo tensor de tímpano e da membrana timpânica ou contração muscular do véu palatino. Tal fato pode provocar disfunção da tuba auditiva e em sequencia, sintomas de plenitude auricular, desequilíbrio e perda de audição $(20,24,26,27)$.

Pode-se dizer que a relação e origem dos sintomas otológicos ainda não foram totalmente esclarecidas (23). Porém, pesquisa (24) comprova que as DTMs, geralmente, vêm associadas a sintomas otológicos.

\section{CONCLUSÃO}

Os achados deste estudo apontam alto percentual de sintomas otológicos em pacientes com DTM. Destaca-se a importância da pesquisa dos sintomas otológicos na avaliação das DTMs. Cabe, ainda, sugestões de outras pesquisas com uma amostra maior de sujeitos para confirmação da relação entre as DTMs e sintomas otológicos. 


\section{REFERÊNCIAS BIBLIOGRÁFICAS}

1. Ilha VCA, Rapoport A, Filho JBI, Reis AA, Boni ASB. Estimativa da excursão condilar em pacientes com disfunção craniomandibular: um enfoque multidisciplinar. Rev Dent Press Ortodon Ortopedi Facial. 2006, 11(3):6370.

2. Silvinelli F, Casale M, PaparoF, Persico AM, Zini C. Subjective tinnitus, temporomandibular joint dysfunction, and serotonin modulation of neural plasticity: causal or causal triad? Med Hypotheses. 2003, 61(4):444-448.

3. Ferrario VF, Tartaglia GM, Luraghi FE, Sforza C. The use of surface electromyography as a tool in differentiating temporomandibular disorders from neck disorders. Man Ther. 2006, 12(4):372-379.

4. Farella MA, Michelotti A, Bocchino T, Cimino R, Laino AMH, Steenks MH. Effects of orthognathic surgery for class III malocclusion on signs and symptoms of temporomandibular disorders and on pressure pain thresholds of the jaw muscles. Int J Maxilofac Oral Surg. 2007, 2(1):101-110.

5. Zocoli R, Mota EM, Sommavilla A, Perin RL. Manifestações otológicas nos distúrbios da articulação temporomandibular. ACM Arq Catarin Med. 2007, 36(1):90-95.

6. Takayama YDMD, Miura PHD, Yuasa DMD, Kobayashi K, Hosoi T. Comparison of occlusal condition and prevalence of bone change in the condyle of patients with and without temporomandibular disorders. Oral Radiol Endod. 2008, 105(1):104-112.

7. Proff P, Richter EJ, Blens T, Fanghanel J, Hutzen D, Korda B et al. A michigan-type occlusal splint with spring-loaded mandibular protrusion functionality for treatment of anterior disk dislocation with reduction. Ann Anat. 2007, 189(4):362366.

8. Barbosa TS, Miyakoda LS, Pocztaruk RL, Gavião MBD. Temporomandibular disorders and bruxism in childhood and adolescence: Review of the literature. Int J Pediatr Otorhinolaryngol. 2008, 72(3):299-314.

9. Castrillon EE, Cairns BE, Ernberg M, Wangd K, Sessle B, Arendt-Nielsen L, Svensson P. Glutamate-evoked jaw muscle pain as a model of persistent myofascial TMD pain? Arch Oral Biol. 2008, 53(7):666-676.

10. Galdón MJ, Durá E, Andreu Y, Ferrando M, Poveda R, Bagán V. Multidimensional approach to the differences between muscular and articular temporomandibular patients:
Coping, distress and pain characteristics. Oral Radiol. 2006, 102(1):40-46.

11. Scolozzi P MDDMD, Becker MMD, Richter MMDDMD. Temporomandibular Joint Osteoarthritis: A Cause of a Serous Otitis Media? A Case Report. J Oral Maxillofac Surg. 2004, 62(1):97-100.

12. Mariz ACR, Campos PSF, Sarmento VA. Avaliação dos deslocamentos de disco da articulação temporomandibular. Braz Oral Res. 2005, 19(1):63-68.

13. Elias FM, Birman EG, Matsuda CK, Oliveira IRS, Jorge WA. Ultrasonographic findings in normal temporomandibular joints. Braz Oral Res. 2006, 20(1):25-32.

14. Siqueira JTT, Ching LH, Nasri C, Siqueira SRDT, Teixeira MJ, Heirs G, Valle LBS. Clinical study of patients with persistent orofacial pain. Arq Neuropsiquiatr. 2004, 62(4):988-996.

15. Bianchini EMG, Paiva G, Andrade CRF de. Movimentos mandibulares na fala: interferência das disfunções temporomandibulares segundo índices de dor. Pró-fono. 2007, 19(1):7-18.

16. Felício CM de, Melchior MO, Silva MAMR, Celeghini RMS. Desempenho mastigatório em adultos relacionado com a desordem temporomandibular e com a oclusão. Pró-fono. 2007, 19(2):151-158.

17. Bianchini EMG. Mandibular movement patterns during speech in subjects with temporomandibular disorders and in asymptomatic individuals. J Craniomandibular Pract. 2008, 26(1):50-58.

18. Machado IM. Bianchini EMG. Andrada e Silva MA. Ferreira LP. Voz e disfunção temporomandibular em professores. Rev Cefac. 2009, 11(4):630-643.

19. Bianchini EMG. Avaliação fonoaudiológica da motricidade oral - Distúrbios miofuncionais ou situações adaptativas. Rev Dent Press Ortodon Ortopedi Facial. 2001, 6(3):73-83.

20. Tuz HH, Onder EM, Kisnisci RS. Prevalence of otologic complaints in patients with temporomandibular disorder. Am J Ortond Dentofacial Orthop. 2003, 123(6):620-3.

21. Keersmaekers KDDS, De Boever JA, Van Den Berghe L. Otalgia in patients with temporomandibular joint disorders. J Odontol Dent. 1996, 75(1):72-76.

22. Parker WS, Chole RA. Tinnitus, vertigo and temporomandibular disorders. Am J Ortond Dentofacial Orthop. 1995, 107(2):153-8. 
23. Felício CM de, Faria TG, Silva MAMR da, Aquino AMCM, Junqueira CA. Desordem Temporomandibular: relações entre sintomas otológicos e orofaciais. Rev Bras Otorrinolaringol. 2004, 70(6):786-793.

24. Mota LAA, Albuquerque KMG, Santos MHP, Travassos RO. Sinais e sintomas associados à otalgia na disfunção temporomandibular. Arq Int Otorrinolaringol. 2007, 11(4):411-415.

25. D'Antonio W E.PA, Ikino CMY, De Castro SM, Balbino APS, Jurado JRP, Bento RF. Distúrbio temporomandibular como causa de otalgia: um estudo clínico. Rev Bras Otorrinolaringol. 2000, 66(1):46-50.
26. Nakashima MMD, Yano HMD, Akita SMD, Tokunaga KMD, Anraku KMD, Tanaka KMD, Hirano AMD. Traumatic Unilateral TemporomandibularJoint Dislocation Overlooked for More Than Two Decades. J Craniofac Surg. 2007, 18(6):1466-1470.

27. Pascoal MIN, Abrão R, Chagas JFS, Pascoal MPBN, Claudiney CC, Magna LA. Prevalência dos sintomas otológicos na desordem temperomandibular: estudo de 126 casos. Rev Bras Otorrinolaringol. 2001, 67(5):627633. 